\title{
Survey on Current Trends and Techniques of Data Mining Research
}

\author{
Prakhar Agarwal ${ }^{1}$, Vinay Pandey ${ }^{2}$, Dr. Bindu Garg ${ }^{3}$ \\ Department of Computer Enginnering ${ }^{1,2,3}$ \\ Bharati Vidyapeeth (Deemed to be University) College of Engineering, Pune ${ }^{1,2,3}$ \\ Email: paprakhar9@gmail.com ${ }^{1}$,vinay95pandey@gmail.com ${ }^{2}$, bindu.garg@bharatividyapeeth.edu ${ }^{3}$
}

\begin{abstract}
The paper studies distinctive parts of information mining research. Information mining is useful in getting information from expansive spaces of databases, information stockrooms and information bazaars. Extraordinary and current zones of information mining moreover talked about. Issues and difficulties of information mining alongside different open source instruments are tended to also. Information mining is an imperative what's more, developing examination territory and utilized by the researcher to analysts and PC researchers too.
\end{abstract}

Keywords- information mining, learning revelation in databases, regions and instruments in information mining, difficulties of information mining.

\section{INTRODUCTION}

Information mining is extricating data and information from colossal measure of information. Information mining is a fundamental advance in finding information from databases. There are quantities of databases, information bazaars, information distribution centers everywhere the world. In the event that the information are not dissected to discover the intriguing examples, at that point the information would become information tombs. Information excavators look for the pearl in the ocean of information. An information mining framework may create loads of examples. Normally a little part of the examples are intriguing. Here the intriguing methods use able, legitimate and novel. In addition, it is practically difficult to remove the intriguing concealed examples with regards to the ocean of information without the assistance of information mining devices. There are seven stages in information mining. They are information cleaning, information reconciliation, information choice, information change, information mining, learning introduction and example evolution[6]. Database innovation had developed from crude document preparing to the improvement of information mining devices and applications. The information might be gathered from different applications including science and building, the executives, business houses, government organization and ecological control. Fascinating information examples might be mined from spatial, time-related, content, natural, interactive media, web and heritage databases. Information mining encourage the executives in basic leadership. The information mining work incorporates the revelation of idea depictions, affiliation, order, forecast, grouping, pattern investigation, deviation investigation and comparability examination. Information mining in vast databases presents different necessities and challenges for the scientists and engineers. A multidimensional information demonstrate is utilized for the plan of information distribution centers and information shops. The center of such model is information block[6]. Information 3D square comprises of expansive arrangement of realities and number of measurements. Measurements are the substances on which an association keeps records. Naturally, they are various leveled.

\section{DIFFERENT AREAS OF DATA MINING \\ 2.1. Web Mining}

As there is colossal measure of information and data accessible in the World Wide Web, the information excavators have a rich zone for web mining. Web mining is information digging strategies for extraction of data from web archives and administrations. The substance of the web are exceptionally unique. It is developing at a fast pace, and the data is consistently refreshed. Web mining might be isolated into the accompanying subtasks[1].

(1) Asset discovering: discovering records expected for the Web.

(2) Data choice and preprocessing: Selection and preprocessing of the data recovered from the Web.

(3) Speculation: To find the general examples from the person just as numerous destinations.

(4) Examination: Discovered examples are deciphered for important information.

Web mining might be separated into Web Structure, Web Contents, and Web Access Patterns.

\subsection{Text Mining}

The term content mining or KDT (Knowledge Revelation in Text) was first proposed by Feldman what's more, Dagan in 1996[1]. The unstructured content may be mined utilizing data recovery, content arrangement, or applying NLP strategies as a preprocessing step. Content Mining includes numerous applications to such an extent that content order, grouping, discovering designs and consecutive 
International Journal of Research in Advent Technology, Vol.7, No.4, April 2019

E-ISSN: 2321-9637

Available online at www.ijrat.org

designs in writings, computational semantics, and affiliation revelation.

\subsection{Spatial Data Mining}

The spatial information mining manages information identified with area. The blast of topographically related information for quick advancement of IT, computerized mapping, remote detecting, GIS requests for creating databases for spatial investigation and demonstrating. Spatial information depiction, order, affiliation, bunching, pattern, and exception investigation are the fundamental parts for spatial information mining.

\subsection{Multimedia Data Mining}

Mixed media information mining investigates the intriguing designs from databases identified with media that deals with a vast gathering of interactive media objects. Mixed media objects incorporate sound, video, picture, arrangement information and hypertext information containing content, content markups, and linkages. Mixed media information inquire about spotlights on content-based recovery, comparability look, affiliation, order and expectation investigation.

\subsection{Time series Data Mining}

A period arrangement database changes its qualities and occasions as for time. A portion of the instances of time arrangement information are securities exchange information, business exchange information, dynamic generation information, therapeutic treatment information, website page get to succession, etc. The time arrangement inquire about includes issues identified with likeness seek, pattern examination, mining successive and intermittent examples in timerelated information.

\subsection{Biological Data Mining}

There is a substantial stockpiling of clinical and natural information from DNA microarray information, genomic arrangements, protein associations just as arrangements, electronic wellbeing records, illness pathways, biomedical pictures and the rundown goes on. In the clinical setting, scholars are endeavoring to discover the organic procedures that are the reason for a ailment. There are a few issues identified with these high-dimensional natural information. These issues incorporate boisterous and deficient information, coordinating different wellsprings of information and preparing PC concentrated assignments. Researcher just as clinical researchers utilized an assortment of information mining instruments to find fascinating and significant perceptions from countless information from diverse organic areas.

\subsection{Education Data Mining}

Instructive Data Mining (EDM) is a developing examine territory worried about the one of a kind sorts of information that originate from instructive settings, and utilizing those techniques to more readily get it understudies. Instructive Data Mining centers around growing new apparatuses and calculations for finding information designs. EDM creates strategies what's more, applies procedures from insights, machine learning, and information mining to break down information gathered amid instructing and learning. New PC bolstered intelligent learning techniques and apparatuses have opened up chances to gather what's more, break down understudy information, to find designs and inclines in those information, and to make new disclosures furthermore, test speculations about how understudies learn. Information gathered from web based learning frameworks can be collected over extensive quantities of understudies and can contain numerous factors that information mining calculations can investigate for model structure. Distinctive understudy models are utilized for expectation of future learning conduct of the understudies. Computational models are utilized dependent on the understudy space and teaching method.

\subsection{Ubiquitous Data Mining (UDM)}

The information diggers have another test in the structure of the pervasive access by utilizing wearable PCs, palmtops, PDAs, PCs. To remove concealed data from these gadgets requires propelled investigation. In the realm of UDM, correspondence, calculation, security, and so forth are a portion of the components. The one of the targets of the UDM is to remove fascinating examples while limiting the extra expense of the processing because of the above-refered to factors. To execute information mining undertakings like arrangement, bunching, affiliations, and so forth are troublesome for pervasive gadgets. Little showcase zones, information the executives in portable are a portion of the difficulties in this respects. The key issues are the propelled calculation for portable and appropriated figuring, information the board issues, information portrayal methods, mix of these gadgets with database applications, UDM design, programming specialists, operator association and uses of UDM[4].

\subsection{Constraint-based Data Mining}

Requirement based information mining is one of the creating regions where the information excavators utilize the imperative for better information mining. One of the utilizations of imperative based information mining is Online Analytical Mining Architecture (OALM) created by[5] and is intended for multidimensional just as imperative based mining in view of databases and information distribution centers. More often than not, information mining procedures need client control. One type of information mining is the place the human contribution is there as imperatives. There are different kinds of imperatives with their claim qualities and reason. They are learning type, information, measurement/level, intriguing quality, rule imperatives.

\section{DATA MINING TOOLS}

Coming up next are the mainstream information mining open source instruments. 
International Journal of Research in Advent Technology, Vol.7, No.4, April 2019

\author{
E-ISSN: 2321-9637 \\ Available online at $w w w . i j r a t . o r g$
}

\subsection{RapidMiner}

This instrument is written in Java programming language, and it offers examination of propelled level through its layout based system. Clients scarcely need to do any coding. RapidMiner is fit for dealing with different assignments like measurable displaying, prescient examination and representation separated from information mining errands. RapidMiner gives learning plans, models and calculations from WEKA and $\mathrm{R}$ contents that make it all the more dominant. This open source is dispersed under the AGPL open source permit and it very well may be downloaded from SourceForge. It is one of the best business investigation programming. Every one of the information mining errands are packaged in one single suite [http://fast i.com/content/see/181/190/].

\section{2. $W E K A$}

Weka was initially created in a non-Java adaptation for investigating horticultural information. Afterward, the Java variant was created, and it turned into a integral asset for various information mining applications like prescient displaying and information investigation. This product is free under the GNU Overall population License, which is a major preferred standpoint contrasted with RapidMiner. As it is free under the GNU General Public License which is a major favorable position of it when contrasted with its partners like RapidMiner. It tends to be redone by the clients. The majority of the information mining occupations are bolstered by Weka. They are arrangement, bunching, relapse, highlight extraction, perception, and so forth. Its graphical UI makes it a better-complex instrument for information mining process. In this way, Weka has turned out to be a standout amongst the most dominant open source information mining programming. [http://en.wikipedia.org/wiki/Weka_(machine_learnin g)] [http://www.cs.waikato.ac.nz/ml/weka/].

\subsection{R-Programming}

Venture R, which is a GNU venture, is written in C, FORTRAN and $\mathrm{R}$ Language. $\mathrm{R}$ language is utilized for composing heaps of modules of the product itself. $\mathrm{R}$ programming is free, and it is likewise utilized for factual registering and illustrations. Information diggers utilized $\mathrm{R}$ for creating measurable bundles what's more, breaking down the information. As of late the prevalence of $\mathrm{R}$ had expanded due to its simplicity of utilization and extensibility. $\mathrm{R}$ gives extraordinary measurable strategies that incorporate straight and nonlinear demonstrating; information mining forms for example order, bunching, time arrangement investigation and others. [http://www.rproject.org/][11].

\subsection{Orange}

Orange, a Python-based, amazing and open source instrument for information digging clients for the reason of learning extraction. It has amazing visual programming and Python scripting appended to it. It tends to be utilized for AI just as bioinformatics and content mining by including addons. It's pressed with highlights for information investigation. Orange has particular additional items like Bioorange for bioinformatics [http://orange.biolab.si/highlights/].

\subsection{KNIME}

KNIME is fit for performing three fundamental assignments in information preprocessing. They are extraction, change, and stacking. The information handling is finished by permitting the gathering of hubs. It is an mix stage with solid information investigation also, revealing. KNIME utilized secluded information pipelining idea for $\mathrm{AI}$ and information mining. It is utilized for business insight too as monetary information mining. KNIME is effectively extendible and can be included a module for explicit employments. This open source is likewise written in Java and in view of Eclipse. The center adaptation comprises of different information joining modules. Its exploration region not just incorporates pharmaceutical research yet in addition business information, budgetary knowledge and CRM client information. [https://en.wikipedia.org/wiki/KNIME].

\subsection{NLTK}

With regards to language preparing errands, NLTK is one of the real players. NLTK is utilized for AI, information mining, supposition investigation and information scratching. It is additionally widely utilized for language preparing. Since it's composed in Python, one can construct applications over it, altering it for little assignments. NLTK played a significant job as a showing apparatus, think about device, prototyping and can be utilized as a stage for great research.

[https://en.wikipedia.org/wiki/Natural_Language_Tool kit]

\section{LITERATURE REVIEW}

There are bunches of data mining considers around the globe.

Understudies Mood acknowledgment[2] was proposed by Christos N. Moridis et. al. for on the web selfevaluation test. Exponential rationale and recipes were utilized in this respects. The sources of info were understudy's past answers and slide bar status. The exponential rationale factors were an aggregate number of inquiries for the online selfassessment test, understudy's objective, and slide bar esteem. Suitable criticisms are recorded based on current status of inclinations of the understudies. Understudy's manual determination of their disposition utilizing slide bar with no computerization is the constraint of the framework.

In[8], the scientists proposed a novel savvy framework which would most likely identify the street mishaps naturally, tell them by utilizing vehicular systems and gauge the seriousness of the mishap dependent on information mining apparatuses and learning impedance. Different factors, for example, the vehicle speed, the kind of vehicles included, the effect speed, and the status of the airbag, and so forth are utilized for estimating the seriousness of the mishap. A model 
International Journal of Research in Advent Technology, Vol.7, No.4, April 2019

E-ISSN: 2321-9637

Available online at www.ijrat.org

dependent on off-the-rack gadgets was created and approved it at the Applus + IDIADA Automotive Research Organization offices, demonstrating that this framework can diminish the time expected to alarm and send crisis benefits strikingly after a mishap takes place. Three characterization calculations were utilized for example, Decision Trees, Support Vector Machines, furthermore, Bayesian systems and were thought about for best outcomes. It was discovered that Bayesian model for order is the most appropriate model.

In[7], the specialists proposed a method for the forecast of what else the client prone to purchase dependent on halfway data about the substance of a shopping basket. The information structure utilized in this setting was itemset trees (ITtrees), they got every one of the principles whose predecessors contain something like one thing that is absent from the shopping basket in a computationally proficient way. The established Bayesian choice hypothesis also, another calculation dependent on Dempster-Shafer (DS) hypothesis of proof mix were consolidated for discovering rules based vulnerability handling procedure. The proposed calculation improved the execution. As the info, the calculation takes an approaching thing set and returns a diagram dependent on affiliation rules involved by the approaching thing set. The proposed calculation utilized profundity first inquiry system and furthermore refreshed the rule chart.

\section{DATA MINING TECHNIQUES}

A few information mining strategies are utilized in information mining errands. Affiliation, grouping, grouping, forecast, consecutive example mining, and so on are information mining procedures.

\subsection{Classification}

Arrangement discovers decides that parcel information into a few gatherings. The contribution for the grouping is the preparing set. The preparation set's class marks are definitely known. Grouping relegates class names to unlabelled records dependent on a model that gets learning from the preparation datasets. Such order is known as directed learning as the class names are known. There are a few order models. A portion of the normal grouping models are choice trees, neural systems, hereditary calculations, support vector machines, Bayesian classifiers. The application incorporates credit chance investigation, extortion identification, banking and therapeutic application, and so on[1].

\subsection{Clustering}

Clustering is a strategy for gathering information so that information inside the group have high comparability and not at all like information in different gatherings. Grouping calculations might be utilized for arranging information, arrange information for model development and information pressure, anomaly location, and so on. Numerous bunching calculations were created and are arranged as apportioning strategies, progressive techniques, thickness based and lattice based techniques. The datasets might be numerical or straight out. K-Means, progressive, DBSCAN, OPTICS, STING are a portion of the outstanding information bunching calculations[10].

\subsection{Association Rule Mining}

Association rule mining is a very much looked into strategy for finding intriguing relations between factors in huge databases. In affiliation rule, the articulation is of the structure $\mathrm{X}=>\mathrm{Y}$, where $\mathrm{X}$ and $\mathrm{Y}$ are set of things[2]. The principle objective is to find every one of the standards that have backing and certainty more prominent than or equivalent to least help or trust in a database. Bolster implies that how regularly $\mathrm{X}$ and $\mathrm{Y}$ happens together as a level of absolute exchanges. Certainty implies that how much a specific thing is subject to another. There is no importance for the examples with low certainty also, support. The clients can extricate helpful and intriguing data from the examples with middle of the road estimations of certainty and backing. The affiliation rule mining calculations incorporate Apriori, AprioriTid, Apriori half and half and Tertius calculations[10].

\subsection{Support Vector Machine}

Support vector machines (SVM) have a place with another class of AI calculations and are in view of measurable learning hypothesis[1]. The principle idea is to non-directly map the informational index into a high dimensional element space and utilize a direct discriminator for order of information. It is fundamentally utilized for relapse, characterization and choice tree development. SVMs select the plane which augments the edge isolating the two classes. The edge is characterized as the separation between the isolating hyperplane to the closest purpose of one, or more the separation from the hyperplane to the closest point in B, where An and B are two straightly distinct sets. SVM has been utilized in numerous applications including face identification, written by hand character and digits acknowledgment, discourse acknowledgment, picture and data recovery[9].

\section{Acknowledgments}

The author communicated his appreciation to Prof. Dr. D. M. Thakore, Head, Department of Computer Engineering, Bharati Vidyapeeth (To be Deemed University), College of Engineering, Pune for his rousing words. The creator likewise recognized Prof. Dr. Bindu Garg, Professor, Department of Computer Engineering for her important proposals.

\section{REFERENCES}

[1] "Data Mining Techniques", University Press, 2013, Arun K Pujari.

[2] "Mood Recognition during Online SelfAssessment Tests" IEEE Transactions on Learning Technologies, VOL.2, NO.1, January March 2009, Christos N. Moridis and Anastasios A. Economies. 
[3] "A Framework for Personal Mobile Commerce Pattern Mining and Prediction", IEEE Transactions on Knowledge and Data Engineering, VOL. 24, NO. 5, MAY 2012, Eric Hsueh-Chan Lu, Wang-Chien Lee, Member, IEEE, and Vincent S. Tseng, Member, IEEE.

[4] "Data Mining to Go: Ubiquitous KDD for Mobile and Distributed Environments", KDD-2001, San Francisco, August 2001, H. Kargupta and A. Joshi.

[5] "Constraint-based, Multidimensional Data Mining", COMPUTER (Special issue on Data Mining), 32(8): 45-50, 1999, J. Han, V.S. Lakshmanan and R T Ng.

[6] "Data Mining: Concepts and Techniques", Morgan Kaufmann Publishers, 2003, Jiawei Han and Micheline Kamber.

[7] "Predicting Missing Items in Shopping Carts", IEEE Transactions on Knowledge And Data Engineering, VOL. 21, JULY 2009, Kasun Wickramaratna, Student Member, IEEE ,Miroslav Kubat, Senior Member, IEEE, and Kamal Premaratne, Senior Member, IEEE.

[8] "A System for Automatic Notification and Severity Estimation of Automotive Accidents", IEEE Transactions on Mobile Computing, VOL.13, NO.5, MAY 2014,Manuel Fogue, Piedad Garrido, Member, IEEE, Francisco J. Martinez, Member, IEEE, Juan-Carlos Cano, Carlos T. Calafate, and Pietro Manzoni, Member, IEEE.

[9] "Pattern Classification Using Neuro Fuzzy and Support Vector Machine (SVM) - A Comparative Study", International Journal of Advanced Research in Computer and Communication Engineering Vol. 2, Issue 5, May 2013, Maya Nayak and Jnana Ranjan Tripathy.

[10] "Data Mining: Techniques, Key Challenges and Approaches for Improvement", International Journal of Advanced Research in Computer Science and Software Engineering, Volume 6, Issue 3, March 2016,N. Mlambo.

[11] "Open-Source Tools for Data Mining in Social Science," Theoretical and Methodological Approaches to Social Sciences and Knowledge Management, pp. 163-176, Paško Konjevoda and Nikola Štambuk. 